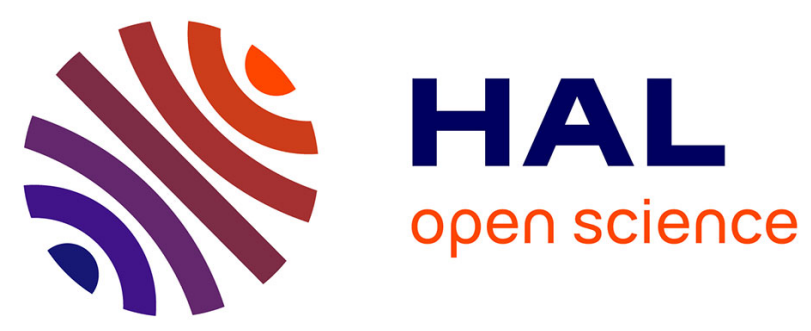

\title{
Myod and H19-Igf2 locus interactions are required for diaphragm formation in the mouse
}

\author{
M. Borensztein, Paul Monnier, Franck Court, Yoann Louault, Marie-Anne \\ Ripoche, Laurent L. Tiret, Zizhen Yao, Stephen J. Tapscott, Thierry Forné, \\ Didier Montarras, et al.
}

\section{To cite this version:}

M. Borensztein, Paul Monnier, Franck Court, Yoann Louault, Marie-Anne Ripoche, et al.. Myod and H19-Igf2 locus interactions are required for diaphragm formation in the mouse. Development (Cambridge, England), 2013, 140 (6), pp.1231-9. 10.1242/dev.084665 . hal-02191602

\section{HAL Id: hal-02191602 https://hal.science/hal-02191602}

Submitted on 28 May 2021

HAL is a multi-disciplinary open access archive for the deposit and dissemination of scientific research documents, whether they are published or not. The documents may come from teaching and research institutions in France or abroad, or from public or private research centers.
L'archive ouverte pluridisciplinaire HAL, est destinée au dépôt et à la diffusion de documents scientifiques de niveau recherche, publiés ou non, émanant des établissements d'enseignement et de recherche français ou étrangers, des laboratoires publics ou privés. 


\title{
Myod and H19-Igf2 locus interactions are required for diaphragm formation in the mouse
}

\author{
Maud Borensztein ${ }^{1}$, Paul Monnier ${ }^{1}$, Franck Court ${ }^{2}$, Yann Louault ${ }^{1}$, Marie-Anne Ripoche ${ }^{1}$, Laurent Tiret ${ }^{3}$, \\ Zizhen $\mathrm{Yao}^{4}$, Stephen J. Tapscott ${ }^{4}$, Thierry Forné2 ${ }^{2}$ Didier Montarras ${ }^{5}$ and Luisa Dandolo ${ }^{1, *}$
}

\begin{abstract}
SUMMARY
The myogenic regulatory factor Myod and insulin-like growth factor 2 (Igf2) have been shown to interact in vitro during myogenic differentiation. In order to understand how they interact in vivo, we produced double-mutant mice lacking both the Myod and Igf2 genes. Surprisingly, these mice display neonatal lethality due to severe diaphragm atrophy. Alteration of diaphragm muscle development occurs as early as $\mathbf{1 5 . 5}$ days post-coitum in the double-mutant embryos and leads to a defect in the terminal differentiation of muscle progenitor cells. A negative-feedback loop was detected between Myod and Igf2 in embryonic muscles. Igf2 belongs to the imprinted H19-Igf2 locus. Molecular analyses show binding of Myod on a mesodermal enhancer (CS9) of the H19 gene. Chromatin conformation capture experiments reveal direct interaction of CS9 with the H19 promoter, leading to increased $H 19$ expression in the presence of Myod. In turn, the non-coding H19 RNA represses Igf2 expression in trans. In addition, Igf2 also negatively regulates Myod expression, possibly by reducing the expression of the Srf transcription factor, a known Myod activator. In conclusion, Igf2 and Myod are tightly co-regulated in skeletal muscles and act in parallel pathways in the diaphragm, where they affect the progression of myogenic differentiation. Igf2 is therefore an essential player in the formation of a functional diaphragm in the absence of Myod.
\end{abstract}

KEY WORDS: Diaphragm differentiation, Genomic imprinting, Myogenesis

\section{INTRODUCTION}

In mammals, several myogenic regulatory factors (MRFs), such as Myf5, Mrf4 (Myf6), Myod and myogenin (Myog), are involved in skeletal muscle development. Myodl (Myod), which was the first gene of this family to be identified, plays an essential role in the determination and differentiation of the skeletal muscle lineage (Pownall et al., 2002; Rudnicki et al., 1993). Mice carrying a targeted deletion of Myod are viable and fertile but they display a growth reduction phenotype of $20 \%$ and deficient muscular regeneration compared with their wild-type (wt) littermates (Megeney et al., 1996; Rudnicki et al., 1992).

Muscles of the diaphragm, as well as limb and tongue, originate from a pool of myogenic migrant precursor cells derived from the hypaxial dermomyotome (Sambasivan and Tajbakhsh, 2007). Under the control of Pax3, these precursors delaminate from the ventrolateral lips of the dermomyotome, migrate and reach the primary diaphragm at around embryonic day (E) 12.5. These cells then express Myod and Myf5; they actively proliferate and fully colonize the diaphragm, with the exception of the tendinous central region. Embryonic and fetal waves of myogenesis induce the production of muscle fibers under the control of Myog to produce a functional diaphragm (Buckingham, 2007).

\footnotetext{
${ }^{1}$ Genetics and Development Department, Inserm U1016, CNRS UMR 8104 University of Paris Descartes, Institut Cochin, 75014 Paris, France. ${ }^{2}$ Institut de Génétique Moléculaire de Montpellier, CNRS UMR 5535, University of Montpellier II, 34293 Montpellier, France. ${ }^{3}$ UMR 955 de Génétique Fonctionnelle et Médicale, Institut National de la Recherche Agronomique, University of Paris-Est, Ecole Nationale Vétérinaire d'Alfort, 94700 Maisons-Alfort, France. ${ }^{4}$ Human Biology Division, Fred Hutchinson Cancer Research Center, Seattle, WA 98109, USA ${ }^{5}$ Molecular Genetics of Development Unit, Department of Developmental Biology, URA CNRS 2578, Institut Pasteur, 75015 Paris, France.
}

*Author for correspondence (luisa.dandolo@inserm.fr)
Interestingly, analysis of mutants of the different MRFs has provided some insight into their importance in the development of the diaphragm. Myod mutants display a delay in hypaxial development that is compensated by the presence of $M y f 5$, as shown by the lethality of Myod;Myf5 double-knockout mutants (Kablar et al., 1998; Kablar et al., 1997). When Myod mutants are bred on an $m d x$ (Dmd) background this also results in death of the newborn pups. They show no alteration of limb muscles but display severe atrophy of the diaphragm (Kablar et al., 2003). In this case, Myf5dependent myogenic precursors are able to compensate for the absence of Myod in the hypaxial limb muscles but not completely in the diaphragm. Myod and Myf5 are both responsible for the establishment and maintenance of the lineage, although they each have specific roles, particularly in the diaphragm. Myog mutants also display neonatal lethality and show a strong reduction in the size of the diaphragm (Hasty et al., 1993; Nabeshima et al., 1993). In this mutant, the precursors are present but their differentiation is severely compromised.

Growth and skeletal muscle development are also controlled by other factors, such as members of the insulin-like growth factor (Igf) family. Igf2 is one of the major growth factors implicated in embryonic growth, cell survival and the differentiation of several tissues (Smith et al., 2006). Mice lacking Igf2 are viable and fertile but display a growth reduction of $40 \%$ compared with their wt littermates (DeChiara et al., 1990). In vitro studies have shown that Igf 2 protein plays a role in myoblast proliferation and differentiation (Rotwein, 2003). Interestingly, a link between the Myod and Igf2 genes was shown in myoblast cell culture (C2 cell line) (Montarras et al., 1996). Further studies suggested that Igf2, through binding to the Igf1 receptor (Igf1r), activates the Akt pathway and Myod downstream targets, although the exact mechanism has not been elucidated (Wilson and Rotwein, 2006; Woelfle et al., 2005). Recently, a microRNA, miR-483-5p, the gene for which is embedded in intron 2 of $\operatorname{Ig} f 2$, has been shown to target the $3^{\prime} \mathrm{UTR}$ 
of serum response factor (Srf) (Qiao et al., 2011). The transcription factor Srf is one of the factors responsible for the activation of Myod expression (Gauthier-Rouviere et al., 1996; L'honore et al., 2003). Nevertheless, little is known in vivo about the role of Igf 2 in skeletal muscle development and about potential interactions between Myod and $\operatorname{Ig} f 2$. Both genes are expressed during embryogenesis, but after birth $\operatorname{Igf} 2$ is strongly downregulated, to be replaced postnatally by Igf1 (Rotwein, 2003).

$\operatorname{Ig} f 2$ belongs to the imprinted H19-Igf2 locus and displays monoallelic expression from the paternally inherited allele. $H 19$ is expressed from the maternal allele and produces a $2.3 \mathrm{~kb}$ noncoding RNA, the function of which has not been fully determined (Gabory et al., 2010; Leighton et al., 1995), as well as a microRNA, miR-675 (Smits et al., 2008). Interestingly, H19 was identified in the same selective screen as Myod and called at the time $\mathrm{MyoH}$ (Davis et al., 1987). The main control element of this locus is the differentially methylated imprinting control region (ICR) located between $I g f 2$ and $H 19$. Both genes are coordinately expressed in many embryonic tissues of mesoderm and endoderm origin. This expression is under the control of two sets of enhancers located downstream of $H 19$ that act as endoderm- or mesoderm-specific enhancers. In addition to cis effects mediated by the enhancers and the ICR, it was recently shown that the $H 19$ non-coding RNA could act in trans by downregulating the expression of $\operatorname{Ig} f 2$ (Gabory et al., 2009; Wilkin et al., 2000).

In order to investigate the link between Igf2 and Myod, we developed a mouse model combining defective alleles for both genes. The double-mutant (DM) mice lacking Myod and Igf2 surprisingly displayed neonatal lethality, whereas the Myod and Igf2 single knockouts were viable. We observed severe atrophy and absence of contraction of the diaphragm, whereas all other muscles displayed no obvious defects. We investigated the mechanisms linked to the non-functionality of the diaphragm in the DM embryos by studying its structural anomalies. Analysis of E13.5-18.5 diaphragms revealed a striking lack of terminal differentiation of the diaphragm of DM embryos. We then studied the molecular pathways linked to Igf2 and Myod and identified a negativefeedback loop between these two genes. H19 expression was also affected by the binding of Myod on the mesodermal enhancers that control this locus. Finally, $S r f$ expression was affected by the lack of Igf2, suggesting a role for this growth factor in the control of Myod expression.

These findings demonstrate a tight interaction between Igf2 and Myod in myogenesis, with the existence of a compensation mechanism by which overexpression of $\operatorname{Ig} f 2$ can compensate for the absence of Myod and vice versa. As shown previously in other contexts, the diaphragm differs strikingly from other skeletal muscles and our results reveal an essential role for $\operatorname{Ig} f 2$, in the absence of Myod, in the production of a functional diaphragm.

\section{MATERIALS AND METHODS}

\section{Mouse strains}

All experimental designs and procedures were in agreement with guidelines of the animal ethics committee of the Ministère de l'Agriculture (France).

$\mathrm{H} 19^{413}$ mice were bred on a C57BL/6 background. $\mathrm{Myod}^{-1}$ mice were bred on a C57BL/6/CBA outbred background and maintained mainly as heterozygotes. The Igf $2^{-/-}$strain was on a $129 / \mathrm{Sv}$ background. Matings were between $\mathrm{Myod}^{+/-}$females and $\mathrm{Myod}^{+/-} ; \mathrm{Igf2}^{+/-}$males, and embryos were collected at E12.5-18.5 (day of plug was considered E0.5).

Matings between a $\mathrm{Myod}^{+/-}$female and a heterozygous $\mathrm{Myod}^{+/-} ; \mathrm{Igf2}^{+/-}$ male produce the four genotypes of interest in the same litter: wild type (wt), $\operatorname{Igf2}^{+/-}$, Myod $^{-/}$and the Myod $^{-/} ; \operatorname{Igf2}^{+/-}$double mutant (DM).
$H 19^{43}$ mice harbor a $3 \mathrm{~kb}$ deletion of the $H 19$ gene and were bred on the 129/Sv background (Ripoche et al., 1997). H19 ${ }^{43}$ females were bred with $\mathrm{Myod}^{-1-}$ males and the resulting heterozygous females were backcrossed to Myod $^{-1}$ males. The resulting progeny were collected at E18.5, genotyped to select $\mathrm{H1}^{-/+} ; \mathrm{Myod}^{+/ /}(n=6)$ and $\mathrm{H1}^{-/+} ; \mathrm{Myod}^{-/+}(n=6)$ embryos and limb muscles and diaphragm were collected.

\section{Genotyping}

DNA was extracted from tail biopsies and PCR was performed with GoTaq polymerase (Promega) according to manufacturer's instructions. Primers are described in supplementary material Table S1.

\section{Embryo collection, muscle histology and immunohistochemistry} E12.5-18.5 embryos were collected from the uterus of $\mathrm{Myod}^{+/-}$and $\mathrm{Myod}^{-/-}$ females and weighed. E18.5 embryos were fixed in 4\% paraformaldehyde, dehydrated and embedded in paraffin. Sections $(5 \mu \mathrm{m})$ were deparaffinized in xylene, rehydrated and stained with Hematoxylin and Eosin (H\&E).

Diaphragms from E13.5, E15.5 and E18.5 embryos were removed and embedded in gelatin-sucrose, frozen in isopentane cooled in liquid nitrogen, and sectioned using a microtome cryostat (Leica). For assessment of tissue morphology, $5 \mu \mathrm{m}$ transverse sections were stained with H\&E.

Fiber size was analyzed by immunostaining muscle sections with an antibody to laminin (Novocastra) and counterstaining with DAPI. Fiber cross-sectional area was determined using MetaMorph software (Molecular Devices).

We analyzed myoblasts and muscle differentiation by immunostaining with Pax7 (Santa Cruz) and myogenin (Dako) antibodies on $7 \mu \mathrm{m}$ transverse sections counterstained with DAPI. The number of positive cells was determined using MetaMorph and ImageJ (NIH) software.

\section{Diaphragm contraction study}

After caesarian delivery at E18.5, newborns were collected and dissected to access the phrenic nerve. Microelectrodes were used to stimulate the phrenic nerve in order to activate the diaphragm with a frequency of $20 \mathrm{~Hz}$, a pulse of 10 milliseconds and 0.5-1.5 V. For wt and $\mathrm{Myod}^{+/}, n=8 ; \operatorname{Igf2^{+/}}, n=1$; Myod $^{-/}, n=2 ; \mathrm{DM}, n=6$.

\section{Electron microscopy}

Electron microscopy was performed on dissected diaphragms from E18.5 wt, single-mutant and DM embryos as previously described (Schmitt et al., 2001).

\section{Whole-mount skeletal staining}

E18.5 fetuses were quickly boiled, skinned and eviscerated. They were fixed in $95 \%$ ethanol for 3 days, and then placed for 24 hours in Alcian Blue solution [15 mg Alcian Blue 8GX (Sigma) in $80 \mathrm{ml} \mathrm{95 \%} \mathrm{ethanol} \mathrm{and} 20 \mathrm{ml}$ glacial acetic acid] at $4{ }^{\circ} \mathrm{C}$ for cartilage staining. Embryos were rinsed for 2 days in $95 \%$ ethanol, then cleared in $1 \% \mathrm{KOH}$ for 2 hours at $4^{\circ} \mathrm{C}$, and counterstained with Alizarin Red solution (5 mg Alizarin Red (Sigma) in $100 \mathrm{ml} 1 \% \mathrm{KOH}$ ) for 3 hours at $4^{\circ} \mathrm{C}$ for bone staining. Embryo clearing was completed in the following ratios of $1 \% \mathrm{KOH}$ to glycerol: 80:20, 60:40, 40:60, 20:80.

\section{ChIP-Seq experiments showing Myod binding}

Myod ChIP-Seq was performed on myoblasts and myotubes in S. Tapscott's laboratory. Results were extracted from published raw data (Cao et al., 2010).

\section{Srf ChIP experiments}

ChIP assays were performed using the HighCell ChIP Kit (Diagenode) with modifications to the manufacturer's instructions to adapt for ChIP on tissues. Limb muscles of wt postnatal day (P) 0 pups were dissected, minced and fixed in $1 \%$ formaldehyde. Tissues were disrupted using a Dounce tissue grinder. Chromatin was sonicated into 200-800 bp fragments using a Bioruptor (Diagenode). Input DNA was purified from $1 \%$ of the chromatin. Total chromatin was used for immunoprecipitation, using protein G-coated magnetic beads (Diagenode) and $2 \mu \mathrm{g}$ Srf antibody (Santa Cruz, SC335 X) or $2 \mu \mathrm{g}$ non-specific rabbit IgG (negative control). DNA was purified and analyzed by quantitative PCR (qPCR). Il4 intron was used as a negative control. Primers are listed in supplementary material Table S1. 


\section{Nuclei extraction from myoblasts}

Limb muscles of E18.5 wt and $\mathrm{Myod}^{-/}$embryos were dissected and dissociated in a solution of $0.75 \mathrm{u} / \mathrm{ml}$ collagenase (Roche), $1.2 \mathrm{u} / \mathrm{ml}$ dispase (Roche), $2.5 \mathrm{mM} \mathrm{CaCl}_{2}$. Collected cells were filtered twice on $70 \mu \mathrm{m}$ and $45 \mu \mathrm{m}$ filters and washed in PBS. Cells were resuspended and lysed in $0.8 \%$ NP40 buffer with $0.3 \mathrm{M}$ sucrose, then loaded on a $1.2 \mathrm{M}$ sucrose solution and centrifuged for 20 minutes at $8500 \mathrm{rpm}(8000 \mathrm{~g})$. Pelleted nuclei were resuspended in $40 \%$ glycerol buffer.

\section{Chromatin conformation capture (3C)}

3C-qPCR experiments were performed on wt and $\mathrm{Myod}^{-/}$nuclei extracted from myoblasts as described previously (Hagège et al., 2007). Interaction frequencies were determined at $\mathrm{BamHI}$ sites surrounding the $H 19$ locus. 3C products were quantified on a LightCycler 480 II apparatus (Roche) and data were normalized according to a published algorithm (Braem et al., 2008). Primers are listed in supplementary material Table S2.

\section{Gene expression analysis}

Collected tissues were disrupted using a MixerMill apparatus (Qiagen) and total RNA was extracted with TRIzol reagent (Invitrogen) according to the manufacturer's instructions. Extracted RNA was RQ1 DNase treated (Promega) and then re-extracted with phenol:chloroform and chloroform before ethanol precipitation.

For the expression profile analysis, reverse transcription with SuperScript II reverse transcriptase (Invitrogen) was carried out on $500 \mathrm{ng}$ total RNA with random hexamer oligonucleotides. Quantitative real-time PCR (RT-qPCR) was performed for Myod, Srf, skeletal actin (Actal) and cardiac actin (Actc1), $\operatorname{Igf} 2$ and $H 19$ on $10 \mathrm{ng}$ cDNA in $10 \mu \mathrm{l}$ final volume with FastStart MasterMix reagent (Roche) in a LightCycler 2.0 apparatus (Roche). The level of gene expression was normalized to the geometric mean of the expression level of Tbp and Gapdh housekeeping genes with geNorm software (v3.4) (Vandesompele et al., 2002). RNA was prepared from five diaphragms and five limb muscles of E18.5 embryos per genotype. Three independent reverse transcription experiments were carried out for each sample.

Detection of miR-483-5p was performed using a stem-loop primer for the reverse transcription step and two primers to detect the level of expression of the microRNA in different samples. Data were normalized to the U6 RNA, using TaqMan microRNA assays according to the manufacturers guidelines (Applied Biosystems).

Primers are listed in supplementary material Table S1.

\section{Statistical analysis}

Data are presented as the mean \pm s.e.m. Statistical significance was determined using a Kruskal-Wallis test followed by post-hoc paired comparisons using Prism software (v5.0a) (GraphPad). $P<0.05$ compared with wt was considered statistically significant.

\section{RESULTS \\ Neonatal lethality and atrophy of the Igf2;Myod double-mutant diaphragm}

In order to study the possible interactions between Igf 2 and Myod in vivo, we produced a mouse model combining defective alleles for both genes. Because $I g f 2$ is imprinted, a paternal heterozygous mouse (Igf $2^{\text {mat }+ \text { pat }}$ - $)$ produces no Igf2 protein. We performed matings between heterozygous or homozygous Myod females $\left(\mathrm{Myod}^{+/-} ; \mathrm{Igf2}^{+/+}\right.$or $\left.\mathrm{Myod}^{-/-} ; \mathrm{Igf}^{+/+}\right)$and heterozygous $\mathrm{Myod}^{+/} ; \mathrm{Igf2}^{+/-}$males to obtain the four genotypes of interest: wt, $\operatorname{Igf2^{+/-}}$, Myod $^{-/-}$and the Myod $^{-/} ;$;gf2 $2^{+/-}$double mutant (DM). Surprisingly, no viable DMs were found in the litters, suggesting embryonic or neonatal lethality of $\mathrm{Myod}^{-/} ; \mathrm{Igf2}^{+/-}$individuals. Embryos collected at different stages showed complete viability of the DMs until the end of fetal development (E18.5) (supplementary material Fig. S1). Embryos of each genotype were weighed between E12.5 and E18.5. In $\operatorname{Igf2^{+/-}}$ mutants, the deficit in weight previously detected at E18.5 $(-40 \%)$, in fact occurs as early as E12.5 $(-20 \%)$ (DeChiara et al., 1990). Myod $^{+/} ;$Igf $2^{+/}$embryos were identical to
$\operatorname{Igf2^{+/}}$ mutants, suggesting that removing one copy of Myod had no synergistic effect on the deleterious Igf2 phenotype. Myod ${ }^{-/}$ embryos displayed a weight phenotype identical to wt embryos. The decrease in weight of $20 \%$ that was previously described for the Myod mutants was detected only after birth (supplementary material Fig. S1). Therefore, it appeared that $I g f 2$ affected the growth of the embryo as early as E12.5, whereas only postnatal growth was affected by the Myod defect. In consequence, the DM embryos display the same weight phenotype as $I g 2^{+/-}$embryos.

Caesarian delivery and genotyping at the end of gestation showed that out of 60 newborns (from eight $\mathrm{Myod}^{-/-}$or $\mathrm{Myod}^{+/-}$mothers), all live pups that survived were either wt, heterozygous or singlemutant pups $(n=48)$, whereas all pups that precociously died were DMs $(n=12)$. DM pups were myotonic, became rapidly cyanotic and exhibited respiratory failure, leading to their death. To evaluate whether they breathed, lungs were collected and immediately placed on a water disk. They sank to the bottom of the tube, which indicated that alveoli had never inflated, in contrast to their healthy littermates (Fig. 1A).

\section{Functional and structural anomalies of the diaphragm of DM embryos}

Experiments were performed to evaluate in vivo diaphragm contraction in E18.5 embryos of the different genotypes. The
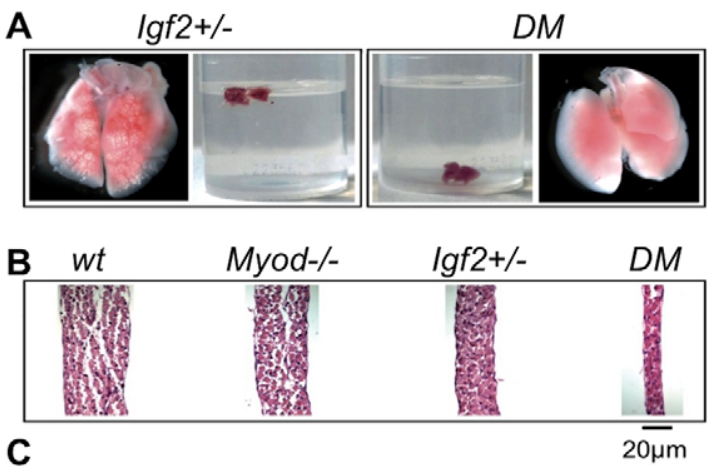

C

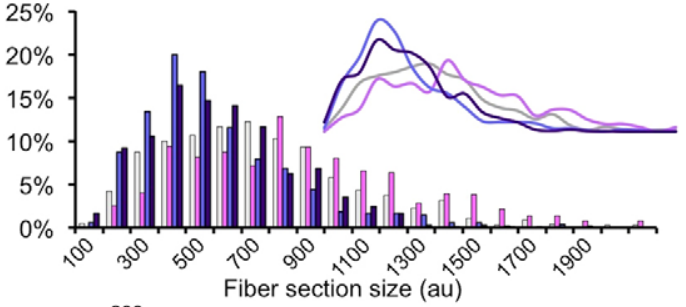

D

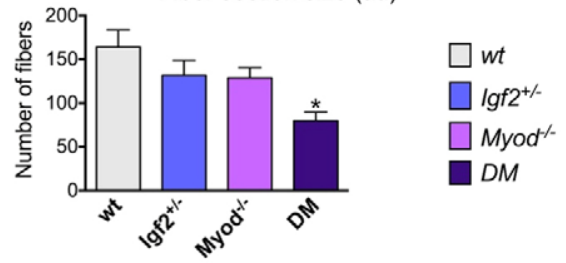

Fig. 1. Diaphragm characteristics in E18.5 mutants. (A) Lungs were dissected out from the mouse embryos after caesarian delivery and dropped into water. $\operatorname{lgf}^{+/-}$lungs float, whereas those from the Myod $^{-/} ; \mathrm{lgf2}{ }^{+/-}$DM sink to the bottom, which means that DM lungs were not inflated with air. (B) Sagittal sections of E18.5 diaphragms stained with H\&E. (C) Immunostaining for laminin on E18.5 diaphragm sections was used to assess the distribution (\%) of fiber cross-sectional area in the four genotypes. au, arbitrary units. The inset illustrates the curve graph of the histogram. (D) E18.5 diaphragm sections immunostained for laminin were used to assess muscle fiber number in the four genotypes. ${ }^{*} P<0.05$; error bars indicate s.e.m. 
phrenic nerves were stimulated through microelectrodes. No contractile reaction was observed in the DM diaphragm in contrast to controls (supplementary material Movie 1). This contraction incapacity could be the major cause of the respiratory insufficiency.

Histological analyses were performed on $\mathrm{H} \& \mathrm{E}$-stained transverse sections from control (wt, $I g f 2^{+/-}$and $\mathrm{Myod}^{-/}$) and DM $\left(\text { Myod }^{-/} ; \operatorname{Igf2^{+/}}\right)^{-1}$ embryos at E18.5 to study skeletal muscles. Unexpectedly, only diaphragms from DM animals showed a substantial size reduction compared with those from controls, whereas DM limb and intercostal muscles presented no obvious phenotype (supplementary material Fig. S2A,B). Atrophy of the DM diaphragm was accompanied by hypoplasia, with a $50 \%$ decrease $(P=0.0128)$ in the number of muscle fibers (Fig. 1B,D). This reduction was specific to the diaphragm, as neither intercostal nor limb muscles presented hypoplasia (supplementary material Fig. S2D). These sections were also used to study the muscle fiber crosssectional area. Comparison between control and DM diaphragms (Fig. 1C) and intercostal muscles (supplementary material Fig. S2C) showed $\sim 50 \%$ reduction in fiber size both in the $I g 2^{+/}$and DM embryos as compared with wt and $\mathrm{Myod}^{-/}$embryos. In summary, the fibers of different skeletal muscles are smaller in the absence of Igf 2 protein. In addition, only the diaphragm displays a reduced number of fibers in the DM embryos.

To pursue the phenotypic description of the DM newborns, we studied the structure of E18.5 diaphragm muscle fibers by electron microscopy. No difference was seen between wt and single-mutant embryos (Igf2 $2^{+/-}$and Myod $^{-/}$) (Fig. 2A-C). By contrast, most of the DM embryo fibers showed sarcomere alignment defects (Fig. 2D). In addition, $\sim 20 \%$ of the fibers presented aberrant Zdisc-like structures, previously described as 'zebra bodies' and occurring rarely in human myopathies (Fig. 2E,F) (Engel and Franzini-Armstrong, 2004; Lake and Wilson, 1975; Nowak et al., 2012).

Finally, we investigated ossification defects in the mutants, as skeletal development is known to be coordinated with muscular development during embryogenesis. Bone and cartilage were compared in E18.5 embryos after staining with Alizarin Red and Alcian Blue (supplementary material Fig. S3). Structures of the forelimb and hind limb digits and of the facial ossification were normal in all embryos. Interestingly, close observation of the sternum showed only four ossification segments in the DM embryos, whereas five segments were normally found in wt and single-mutant embryos. However, this anomaly was only observed in four out of five DM embryos, suggesting an incomplete penetrance of the phenotype. Since wt E17.5 embryos display only four ossification segments (Kaufman, 1992), some DM embryos might suffer from delayed development of the ossification structure of the sternum. Because of the incomplete penetrance, this anomaly was not considered a major element of the lethality phenotype.

\section{Effects of Myod and Igf 2 on the secondary wave of myogenesis}

In order to understand the phenotypic defects occurring in the diaphragm of the DM embryos, its structure was analyzed during embryogenesis. Myogenic precursors colonize the diaphragm and form primary fibers between E12 and E14.5. The secondary wave of myogenesis (fetal wave), resulting in terminal differentiation of the myotubes, begins at E14.5 (Buckingham, 2007; Sambasivan and Tajbakhsh, 2007). We first determined the size of the diaphragm at E13.5, E15.5 and E18.5 in the various mutant embryos ( $n=3-10$ for each stage and each genotype). Immunostaining for laminin, a marker for myofiber membranes used to delimit skeletal fiber shape, revealed no difference in fiber number of the diaphragm at E13.5 between the wt, single-mutant and DM embryos (supplementary material Fig. S4A). Immunostaining for Pax7, a marker of early myoblasts, showed that the number of Pax7-positive cells was similar between control and DM E13.5 embryos (supplementary material Fig. S4A). By contrast, at E15.5, whereas wt and $I g \mathrm{f}^{+/}$ diaphragms were similar, both $\mathrm{Myod}^{-/}$and DM diaphragms were thinner (reduced by 60\%,P<0.01). $\mathrm{Myod}^{--}$and DM diaphragms also displayed a 3 -fold increase $(P=0.0275)$ in the number of Pax7positive cells, with respect to the number of fibers (Fig. 3A-C).

The secondary wave of myogenesis is an important period for diaphragm formation and atrophy appears as early as E15.5. Thus, we targeted this time point to study diaphragm differentiation. Myog is one of the main factors controlling terminal muscle differentiation and E15.5 sections were immunostained for this transcription factor (Fig. 3E). Strikingly, a 60\% reduction $(P=0.0024)$ in the number of Myog-positive cells was seen in the DM (Fig. 3F).

At E18.5, the $\mathrm{Myod}^{-/}$diaphragm was, surprisingly, of the same thickness as the wt diaphragm, showing that a rescue mechanism operated by the end of gestation (Fig. 1; supplementary material Fig. S4B). By contrast, the DM diaphragm remained thinner than that of the other genotypes, with a 4-fold increase $(P<0.0001)$ in Pax7-positive cell number (Fig. 3D; supplementary material Fig. S4B). In the absence of both Myod and $\operatorname{Igf2}$, differentiation is therefore severely impaired in the diaphragm during the secondary wave of myogenesis.

\section{A negative-feedback loop between Igf2 and Myod} Single Myod or Igf2 mutants are viable and display no skeletal muscle phenotype, whereas the double deletion of these genes leads
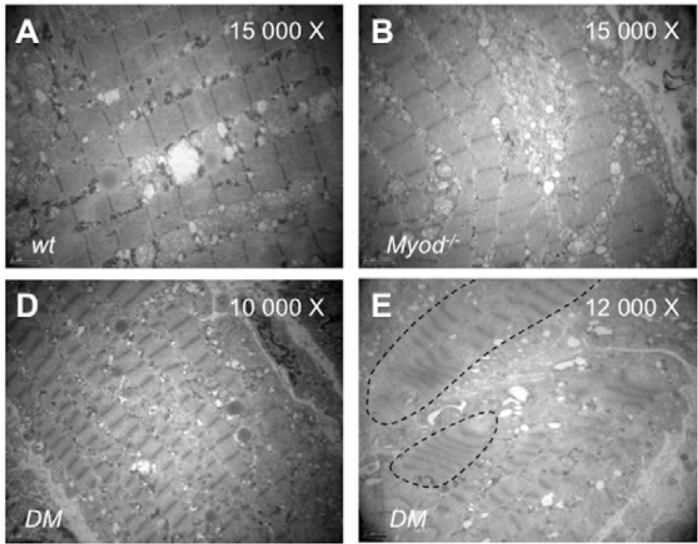
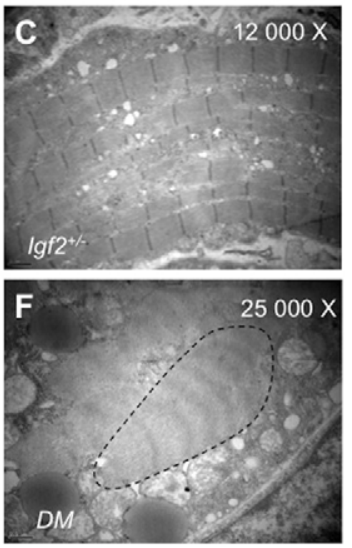

Fig. 2. Electron microscopy of the mouse diaphragm. Representative micrographs showing ultrastructural details of the diaphragm of each genotype. (A-C) wt and single mutants show no anomalies of the sarcomeres. (D-F) Examples of abnormal sarcomeres and of zebra bodies (dashed outlines in E and F) in DM. 

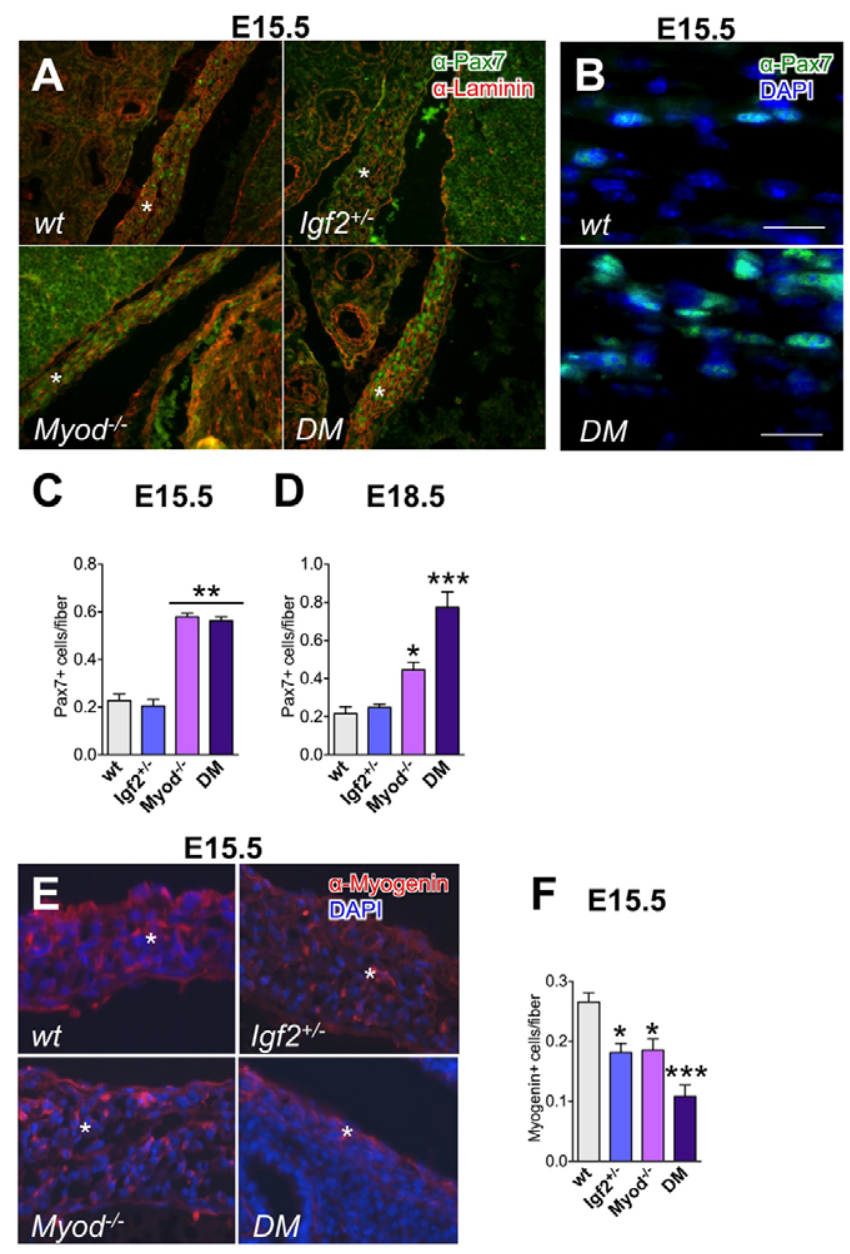

Fig. 3. Diaphragm formation during mouse development. (A) Sagittal sections of E15.5 wt, lgf2 ${ }^{+/}$, Myod $^{-/-}$and DM embryos were immunostained for Pax7 (a-Pax7, green) and laminin (a-Laminin, red). Myod $^{-/-}$and DM diaphragms are thinner than those of wt and $/ \mathrm{gf}^{+/-}$ Stars indicate the position of the diaphragm. (B) Higher magnification of Pax7-stained wt and DM diaphragms. Scale bars: $25 \mu \mathrm{m}$. (C) The number of Pax7-positive cells is increased (3-fold) in $\mathrm{Myod}^{-/-}$and DM diaphragms compared with wt and $/ g f 2^{+/-}$. (D) At E18.5, DM diaphragms continue to present a strong increase (4-fold) of Pax7-positive cells. (E) Sagittal sections of E15.5 wt, lgf2 ${ }^{+/-}$, Myod ${ }^{-/-}$and DM embryos immunostained for Myog (a-Myogenin, red) and counterstained with DAPI (blue). Stars indicate the position of the diaphragm. (F) E15.5 DM embryos present a severe decrease (60\%) in the number of Myog-positive cells in diaphragm compared with wt. ${ }^{*} P<0.05,{ }^{* *} P<0.01,{ }^{* *} P<0.001$; error bars indicate s.e.m.

to neonatal lethality due to diaphragm atrophy. This phenotype could be explained by a redundancy of Myod and Igf 2 in diaphragm formation and promotes the hypothesis of a compensatory mechanism between these two genes. To characterize the differential regulatory interactions between $\operatorname{Myod}$ and $\operatorname{Igf2}$, we performed RT-qPCR on mRNAs from diaphragm and limb muscles of E18.5 control embryos (wt, Myod $^{-/}$and $\operatorname{Igf} 2^{+/-}$). The level of expression of Igf2 was significantly increased in $\mathrm{Myod}^{-/-}$samples (2.5-fold; $P=0.0028$ and $P=0.0025$, respectively, in diaphragm and limb muscles) compared with wt (Fig. 4A) and could compensate for the absence of Myod during diaphragm development. This suggests that Myod can negatively regulate $\operatorname{Ig} 22$ expression. The
A

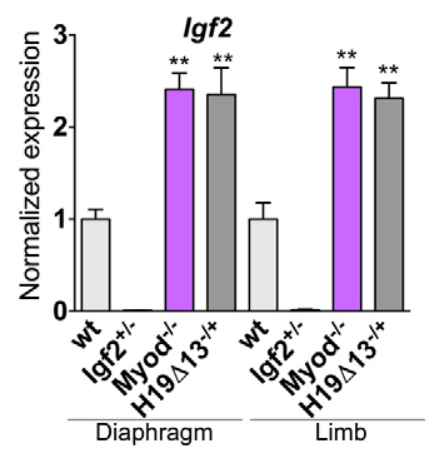

B

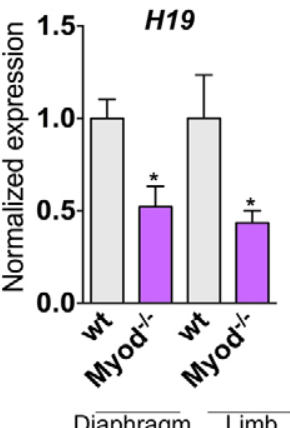

C
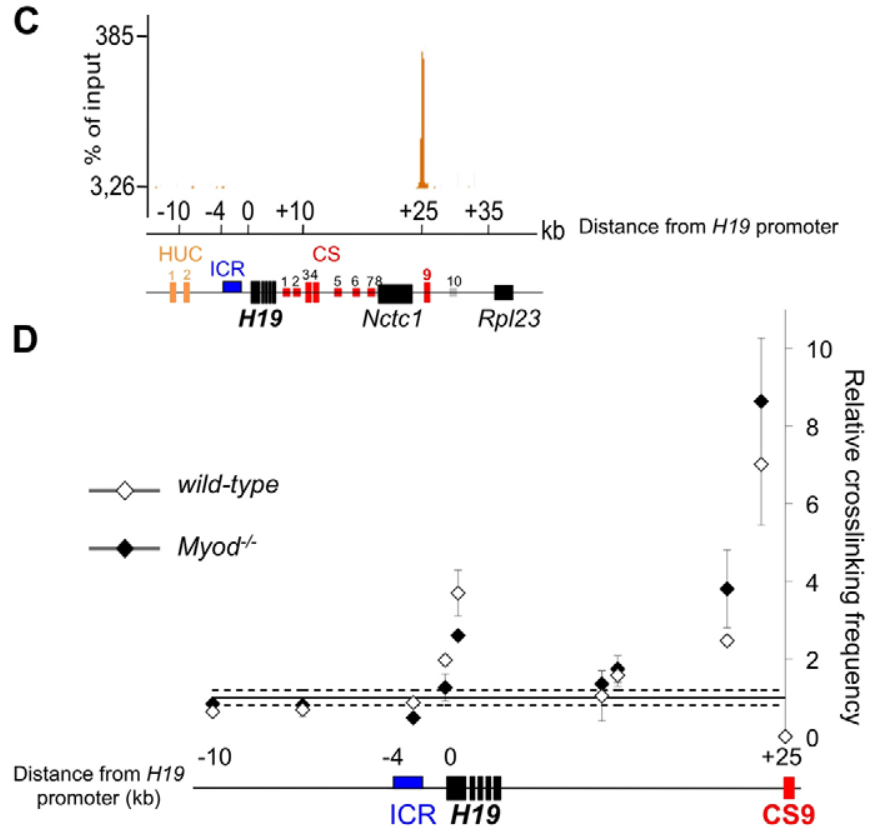

Fig. 4. Myod interaction with the H19-lgf2 locus. (A) The expression level of Igf2 mRNA as assessed by RT-qPCR in diaphragm and limb muscle samples from wt, Myod ${ }^{-/-}$, Igf2 ${ }^{+/-}$and $H_{1} 9^{413-/+}$ mouse embryos. (B) Expression level of H19 RNA assessed by RT-qPCR in diaphragm and limb muscles from wt and Myod $^{-1-}$ embryos. (C) ChIP-Seq data showing the position of the peak of Myod binding in the H19 locus. The genes in this region are indicated by black boxes. Red boxes indicate the endodermal and mesodermal enhancers described in the literature. ICR, imprinting control region; HUC, H19 upstream conserved region; CS, conserved sequence. (D) 3 C experiment showing the interaction between the mesodermal enhancer CS9 (located at $+25 \mathrm{~kb}$ relative to the start of the H19 gene) and the H19 promoter. The location of the ICR, H19 gene and CS9 enhancer are shown by rectangles. ${ }^{*} P<0.05$, ${ }^{*} P<0.01$; error bars indicate s.e.m.

level of expression of Myod was also strongly increased (over 10fold, $P=0.003$ ) in $I g f 2^{+/-}$compared with wt embryos (Fig. 5A). These results reflect a compensatory mechanism in the absence of Igf2.

As an additional control, we included samples from $H 19^{413}$ embryos. These mutants carry a $13 \mathrm{~kb}$ deletion of the $H 19$ gene and its ICR and are known to produce a double dose of Igf 2 mRNA (Fig. 4A) (Leighton et al., 1995). In H19 113 embryos, Myod expression was halved $(P=0.0177)$ compared with wt samples, confirming the control by Igf2 of Myod expression (Fig. 5A).

In conclusion, this is the first evidence of a specific negativefeedback loop between Myod and Igf 2 in mouse embryonic muscles. 


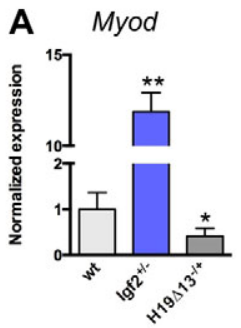

$\mathbf{F}$

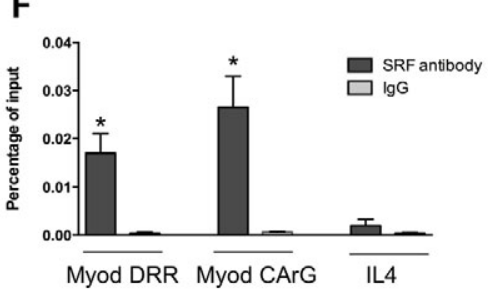

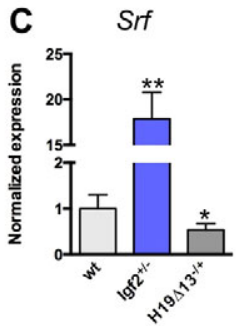
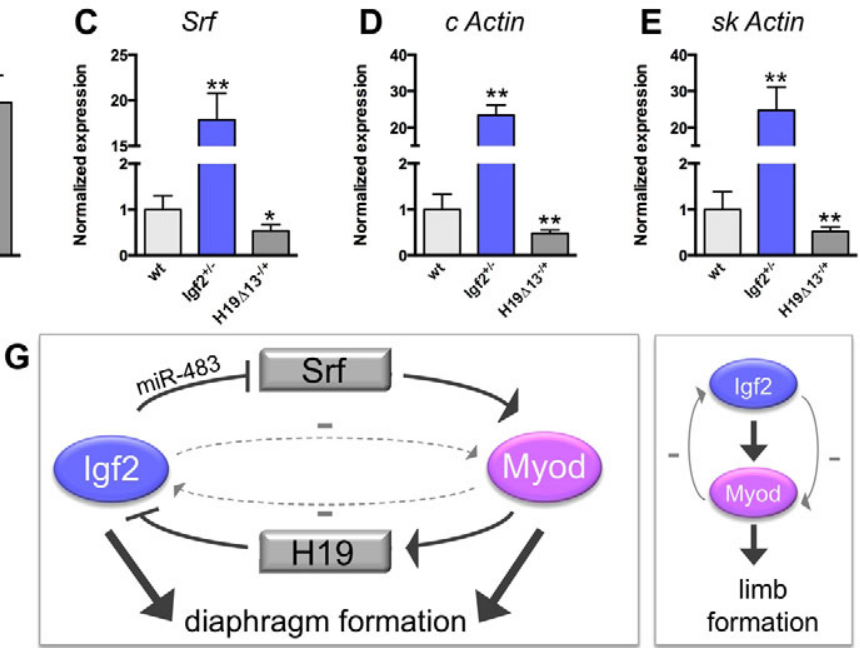

Fig. 5. Control of Myod expression by Igf2. (A-E) Expression level of Myod, miR-483-5p, Srf, cardiac actin (c Actin) and skeletal actin (sk Actin) mRNA in limb muscle from wt, Igf2 ${ }^{+/-}$and $H 19^{413-/+}$ mouse embryos. (F) Srf ChIP assay in limb muscles extracted from wt newborns (P0) on the Myod distal regulatory region (DRR) including the CArG element. Values indicate the percentage of the input with the Srf antibody compared with normal IgG. II4 is a negative control. ${ }^{*} P<0.05$, ${ }^{*} P<0.01$; error bars indicate s.e.m. (G) The negative-feedback loop (dashed arrows) postulated to occur between lgf2 and Myod via H19 and miR-483-Srf in diaphragm and limb muscles. The production of miR-483 by the Igf2 gene negatively controls the Srf transcript level. Srf is known to control Myod expression. In turn, Myod downregulates the Igf2 transcript level through activation of the non-coding H19 RNA. Myod and Igf2 act in two different pathways during diaphragm formation, whereas Igf2 acts upstream of Myod during limb development.

\section{Control of Igf2 expression and molecular interactions at the H19-Igf2 locus}

$H 19$ and Igf2 display highly coordinated regulation in expression, as they are expressed in the same endoderm- and mesoderm-derived tissues throughout development. We analyzed the level of expression of H19 RNA in the different mutant embryos. H19 RNA was reduced by half in $\mathrm{Myod}^{-1}$ muscles compared with wt muscles ( $P=0.0232$ for diaphragm and $P=0.0357$ for limb muscle) (Fig. 4B), suggesting that Myod promotes $H 19$ gene expression in skeletal muscle.

We then investigated the function of the mesodermal enhancers located downstream of the H19 gene by two approaches. First, the interaction of the Myod transcription factor with these mesodermal enhancers was investigated by ChIP-Seq, based on the results obtained by Cao et al. (Cao et al., 2010). Second, we performed a chromatin conformation capture $(3 \mathrm{C})$ experiment in myoblasts extracted from limb muscle to study the interactions of these enhancers with the regulatory elements of the locus.

Several mesodermal enhancers have been identified in the region downstream of the H19 gene (Davies et al., 2002; Ishihara et al., 2000; Kaffer et al., 2001; Yoon et al., 2007). ChIP-Seq data revealed strong binding of Myod in this downstream region (Fig. 4C). This observation emphasizes the functional use of the CS9 enhancer in muscle (Alzhanov et al., 2010; Ishihara et al., 2000). Another binding site for Myod was detected in a region close to the Igf2 gene, but only present in myotubes (supplementary material Fig. S5B).

Next, we collected wt and $\mathrm{Myod}^{-/}$myoblasts from limb muscles (from E18.5 embryos) and performed a quantitative 3C experiment on these cells. Because of the large number of cells $\left(4 \times 10^{7}\right)$ required for this experiment, we chose to use myoblasts from limb muscle rather than from diaphragm. The data clearly show a strong interaction of the CS9 mesodermal enhancer with the promoter of the $H 19$ gene in wt myoblasts (Fig. 4D). As shown previously (Yoon et al., 2007), the maternal mesodermal enhancer interacts with the maternal $H 19$ promoter. In $\mathrm{Myod}^{--}$myoblasts, the interaction of CS9 with the $H 19$ promoter was strongly downregulated ( $P=0.02)$ (Fig. 4D). Therefore, Myod seems to stabilize this interaction.

This CS9 enhancer-H19 promoter interaction provides a link between Myod association and the H19-Igf2 gene regulation. In order to investigate whether Myod has a direct or indirect effect on Igf2 expression, we produced $\mathrm{H}^{-9^{-/+}}$; $\mathrm{Myod}^{-/}$and $\mathrm{H}^{-9^{-/}}$; $\mathrm{Myod}^{+/-}$ E18.5 embryos. For this experiment, we used the $H 19^{\lrcorner 3}$ mutants, in which the ICR is present, in order to maintain the interaction between the mesodermal enhancer and the H19 regulatory sequences. The level of expression of Igf2 was fully comparable in all samples in the absence or presence of Myod (supplementary material Fig. S5A). This indicates that the negative regulation by Myod of Igf 2 expression is not observed in the absence of the H19 gene.

In summary, our data suggest that the binding of Myod to a mesodermal enhancer of the H19-Igf2 locus activates the transcription of H19. However, as H19 RNA is still expressed, although at a lower level, in $\mathrm{Myod}^{-/}$muscle, Myod is not its only activator. The H19 non-coding RNA, by an as yet unknown mechanism, downregulates Igf2 expression by acting in trans, as we and others have previously shown (Gabory et al., 2009; Wilkin et al., 2000). Finally, the negative control exerted by Myod on Igf2 expression is mediated by the $H 19$ gene.

\section{Control by Igf2 of Myod expression}

Since we observed that $\operatorname{Igf} 2$ also exerts negative control on Myod expression to compensate its own absence, we investigated the molecular mechanism underlying this regulation by analyzing the level of expression of miR-483-5p produced by the Igf2 gene. We first showed that this microRNA is normally expressed in wt embryonic muscles (Fig. 5B). In $H 19^{413}$ muscles, which present a double dose of $I g f 2$ transcripts, miR-483-5p expression is increased 2 -fold $(P=0.0159)$ compared with wt samples. 
Srf was recently shown to be a direct target of miR-483 (Qiao et al., 2011) and is known to be a direct regulator of Myod expression. We confirmed by ChIP assay on whole limb muscle tissue that Srf binds to the Myod CArG box (Fig. 5F). We then analyzed Srf expression levels, as well as those of its associated targets, the cardiac and skeletal $\alpha$-actins, in wt, $\operatorname{Ig} f 2^{+/-}$and $H 19^{413}$ muscles (Fig. 5C-E). All three mRNAs were increased 15- to 20-fold $(P=0.0048, P=0.0033$ and $P=0.0033)$ in the absence of $\operatorname{Ig} f 2$ and reduced by half $(P=0.0159, P=0.0095$ and $P=0.0075)$ in the $\operatorname{Ig} f 2$ overexpressing $H 19^{413}$ muscles, compared with wt levels. With these data, and because Srf is one of the main transcription factors controlling Myod expression in muscle (Gauthier-Rouviere et al., 1996; L'honore et al., 2003), we propose as a working hypothesis that Igf 2 controls Myod expression by downregulating Srf, possibly via the production of miR-483.

\section{DISCUSSION}

Our aim in this study was to investigate in vivo the interactions between the Myod and Igf2 genes. Myod $^{-1}$ and $\operatorname{Igf} 2^{+/ p a t-}$ single mutants are viable although they both show a reduction in size. The non-viability of the DMs was surprising and this led us to investigate in detail the timing and cause of death. After collecting the embryos at E18.5, it became obvious that although the newborns were myotonic, they quickly died of respiratory failure. Atrophy of the diaphragm due to a reduced number of muscle fibers and lack of contraction was the probable cause of death. The contraction insufficiency of the DM diaphragm can be linked to the defect in sarcomere alignment and to the presence of 'zebra bodies'. Close examination of the $\mathrm{Myod}^{-/} ; \mathrm{Igf2}^{+/-}$newborns showed no other muscular defects.

Several conclusions can be drawn from these results. Either the lack of both $\operatorname{Igf} 2$ and Myod is compensated in limb and intercostal muscles but not in the diaphragm, or $I g f 2$ expression is not essential for normal development of the limb and intercostal muscles. Alternatively, because a functional diaphragm is an absolute requirement at birth, any alteration in its function will lead to neonatal lethality. In any case, our data strongly suggest that, in the absence of Myod, Igf2 is essential for functional development of the diaphragm. These two genes appear to play a major role during late embryogenesis, confirming previous data showing that $\operatorname{Ig} f 2$ is involved in terminal differentiation (Merrick et al., 2007). Because the effect of the double mutation is more severe than either single mutation, it can be concluded from our data that the $\operatorname{Igf} 2$ and Myod genes act in parallel independent pathways in the diaphragm (Fig. 5G).

Although limb and diaphragm muscles both originate from hypaxial migratory precursors, a number of differences exist between the limb musculature and diaphragm, as evidenced by the fact that in muscular dystrophies the diaphragm is more severely affected than the limb muscles. Defective diaphragm phenotypes have been described previously in $\mathrm{Myog}^{-/-}$and $\mathrm{Myod}^{-/-} ; \mathrm{mdx}$ mutants, suggesting that these two MRF genes play an essential role in diaphragm formation (Hasty et al., 1993; Inanlou et al., 2003). The study of the $\mathrm{Myod}^{-1-} ; m d x$ mutants revealed that the primary myoblasts, which are constantly produced in the $m d x$ mutants, proliferate but are unable to differentiate in the diaphragm. This resulted in an increase in undifferentiated myoblasts. It is also known that whereas Myf5 is capable of compensating the absence of Myod in most muscles, it is unable to fully perform this function in the diaphragm, suggesting that this organ is more sensitive to modulations in the expression of MRFs. Finally, it was recently shown that in double-null Notch signaling/Myod mutants, the diaphragm displays a dramatic phenotype compared with limb muscles (Bröhl et al., 2012). Limb and intercostal muscles in the DM pups do not display any particular defect at birth because of the redundancy with the Myf5 gene, whereas the diaphragm is severely affected.

To further investigate the altered function of the $\mathrm{Myod}^{-/} ; \operatorname{Igf2}^{+/-}$ diaphragm, we analyzed its formation during the embryonic and fetal waves of myogenesis. No difference was observed at E13.5 between wt, Myod $^{-/-}$and Igf2 $2^{+/}$single mutants and DM embryos. However, at E15.5, lack of Myod leads to a thinner diaphragm, with the decrease of Myog expression and an increase in the number of Pax7-positive myoblasts. Our results emphasize the observation of a delay in hypaxial myogenesis in the absence of Myod. Interestingly, at term (E18.5), the $\mathrm{Myod}^{-/}$diaphragm is functional and of similar size to that of the wt, but still displays an increase in myoblast number, as shown previously (Inanlou and Kablar, 2003; Macharia et al., 2010). The E15.5 DM diaphragm phenocopies the $\mathrm{Myod}^{-1}$ diaphragm in terms of size and myoblast number. However, at E18.5, unlike the $\mathrm{Myod}^{-/}$mutants, DM embryos maintain an extremely low number of Myog-positive cells and a high number of Pax7-positive cells. These observations confirm a severe defect in the differentiation capacity of the myoblasts in the DM diaphragm and cannot be fully explained by a delay, as in Myod $^{-/}$diaphragm development, as evidenced by the specific anomalies of these diaphragms (sarcomere alignment defects, zebra bodies). The combined absence of Myod and Igf 2 therefore leads to a defective secondary myogenesis, which explains the diaphragm atrophy at birth.

Myog knockout embryos display severe, lethal phenotypes, including a defective diaphragm with abnormal sarcomeres and a delay in ossification. These phenotypes appear at a reduced level in the Myod $^{-/} ;$Igf $^{+/-}$DM embryos. Our results suggest that Igf 2 and Myod act through independent pathways to activate terminal differentiation in the diaphragm via Myog. This leads to a phenotype that is less drastic than, but similar to, that of Myog mutants. In support of this hypothesis, activation of Myog by Igf2 via the Igfbp5 protein has recently been postulated in vitro (Ren et al., 2008). This activation is independent of Myod.

In conclusion, it has been shown in previously described mutants that Myod and Myog play important roles in diaphragm formation. Our model shows that Igf2 is also essential for diaphragm development by affecting the differentiation capacity of primary myoblasts in the absence of Myod.

Our investigation of molecular interactions between Myod and Igf2 implies the existence of a negative-feedback loop between these two genes, both in diaphragm and limb muscle. Myod overexpression compensates for $\operatorname{Igf} 2$ knockout and $\operatorname{Ig} f 2$ overexpression compensates for Myod knockout as a result of this reciprocal negative regulation.

The atrophy in the diaphragm of DM embryos reflects its greater sensitivity to MRF variations as compared with other skeletal muscles. Interestingly, a positive-feedback loop between the Myod and $\operatorname{Ig} f 2$ genes has been described in muscle cell culture (Montarras et al., 1996). The same authors recently described a striking difference in the expression of $\operatorname{Ig} f 2$ between activated satellite cells collected in vivo and satellite cells maintained in culture (Pallafacchina et al., 2010). This suggests that molecular interactions could differ substantially between in vitro and in vivo models.

$\operatorname{Igf2}$ belongs to an imprinted locus and its expression is strongly coordinated with that of the non-coding H19 RNA throughout embryogenesis. When we discovered that Myod could bind to one 
of the mesodermal enhancers of the locus, we further investigated the dynamics of this region. Many putative mesodermal enhancers of $H 19$ have been identified over the years (Davies et al., 2002; Ishihara et al., 2000; Kaffer et al., 2001). More recently, through studies of multiple mouse mutants or using Myod-induced differentiation of cultured mesenchymal cells, the region defined as CS9 was confirmed to be a mesodermal enhancer (Yoon et al., 2007; Alzhanov et al., 2010). Our quantitative 3C data confirm strong interactions of the CS9 sequence with the H19 gene promoter and reinforce the functionality of this mesodermal enhancer. In $\mathrm{Myod}^{-/}$ myoblasts, we were able to show a much weaker interaction between CS9 and the $H 19$ promoter and a reduced expression of the $H 19$ gene. We can therefore postulate that expression of $H 19$ is under the control of Myod in muscle cells. However, since H19 remains expressed, although at a lower level, Myod is clearly not the only activator of the $\mathrm{H} 19$ gene. We also observed an interaction between the CS9 enhancer and the Igf2 promoters (supplementary material Fig. S5B), but we assume that this interaction has a minor effect because $I g f 2$ expression, as shown in double-mutant $H 19^{43}$ $\mathrm{Myod}^{+}$and H1943 $\mathrm{Myod}^{-}$tissues, is controlled by H19. We had previously shown, using both gain- and loss-of-function $H 19$ mutant mice, that the $H 19$ non-coding RNA controls the level of expression of Igf2 in embryonic muscle in trans (Gabory et al., 2009). The negative control by Myod of Igf2 expression is therefore exerted via the enhanced expression of the $H 19$ gene. In conclusion, the Myod transcription factor appears to be a key regulator of H19 expression in muscle. In vivo, Myod binding on the CS9 enhancer results in reduced expression of the $I g f 2$ gene via $H 19$.

Although the involvement of $\operatorname{Igf} 2$ in myogenesis has been extensively studied, its exact function has not been elucidated. It has been shown that Igf2 acts on Myod by binding to the Igf1 receptor and activating the PI3K/Akt pathway in cultured myoblasts (Wilson and Rotwein, 2006). It is also known that Igf2 enables Myod to activate its target genes by acting on the recruitment of Myod co-activators (Polesskaya et al., 2001). More recently, the Srf transcription factor has been shown to be a direct target of miR483-5p, the gene for which is embedded in intron 2 of Igf2 (Qiao et al., 2011). Srf is one of the factors responsible for the activation of Myod expression. We hypothesized that the Igf 2 gene could control Myod expression via miR-483. Interestingly, we were able to show that Srf and its direct targets are upregulated in the absence of this microRNA and are downregulated in the presence of a double dose of the microRNA in $H 19^{413}$ muscles. Our results lead to the conclusion that $\operatorname{Ig} 2$ has a repressive effect on Myod expression via miR-483 and that Srf might be an intermediary of this negative transcriptional control.

In summary, our study of $\mathrm{Myod}^{-/} ; \operatorname{Igf}^{+/-}$embryos has shown (1) the presence of a defective diaphragm that results in lethality of newborn pups, (2) that $I g f 2$ appears to be an essential component for correct diaphragm development in the absence of Myod during the fetal myogenic wave and (3) that the H19-Igf2 locus seems to play an essential regulatory role in muscle differentiation via Myod control.

\section{Acknowledgements}

We thank E. Robertson and A. Efstratiadis for lgf2 $2^{-/-}$mice; M. Rudnicki for $\mathrm{Myod}^{-1-}$ mice; S. Tilghman for the use of the $\mathrm{H}^{19^{113}}$ mice; D. Daegelen, P. Maire, F. Legrand, A. Sotiropoulos and members of their group for invaluable help and advice; S. Tajbakhsh for constructive discussions during the course of this study; F. Pilot-Storck and M. Maurer for help in the assessment of diaphragm contraction; V. Ea for help with the $3 C$ experiments; X. Gao for advice on the microRNA assays; A. Schmitt, D. Couton and C. Godard for help with electron microscopy and histology experiments; and J. Barat for help with graphical design

\section{Funding}

This work was supported by funding from the Association Française contre les Myopathies to L.D.; the Centronuclear Myopathy (CNM) Project to L.T.; the Agence Nationale de la Recherche (ANR) Epinet Project to T.F. and L.D.; the Association pour la Recherche contre le Cancer to L.D. and T.F.; as well as fellowships from the Ministère de la Recherche et Technologie and the Fondation de la Recherche Médicale to M.B. and from the Ligue Nationale contre le Cancer to F.C.

\section{Competing interests statement}

The authors declare no competing financial interests.

\section{Supplementary material}

Supplementary material available online at

http://dev.biologists.org/lookup/suppl/doi:10.1242/dev.084665/-/DC1

\section{References}

Alzhanov, D. T., Mclnerney, S. F. and Rotwein, P. (2010). Long range interactions regulate lgf2 gene transcription during skeletal muscle differentiation. J. Biol. Chem. 285, 38969-38977.

Braem, C., Recolin, B., Rancourt, R. C., Angiolini, C., Barthès, P., Branchu, P. Court, F., Cathala, G., Ferguson-Smith, A. C. and Forné, T. (2008). Genomic matrix attachment region and chromosome conformation capture quantitative real time PCR assays identify novel putative regulatory elements at the imprinted DIk1/Gt12 locus. J. Biol. Chem. 283, 18612-18620.

Bröhl, D., Vasyutina, E., Czajkowski, M. T., Griger, J., Rassek, C., Rahn, H. P., Purfürst, B., Wende, H. and Birchmeier, C. (2012). Colonization of the satellite cell niche by skeletal muscle progenitor cells depends on Notch signals. Dev. Cell 23, 469-481.

Buckingham, M. (2007). Skeletal muscle progenitor cells and the role of Pax genes. C. R. Biol. 330, 530-533.

Cao, Y., Yao, Z., Sarkar, D., Lawrence, M., Sanchez, G. J., Parker, M. H., MacQuarrie, K. L., Davison, J., Morgan, M. T., Ruzzo, W. L. et al. (2010). Genome-wide MyoD binding in skeletal muscle cells: a potential for broad cellular reprogramming. Dev. Cell 18, 662-674.

Davies, K., Bowden, L., Smith, P., Dean, W., Hill, D., Furuumi, H., Sasaki, H., Cattanach, B. and Reik, W. (2002). Disruption of mesodermal enhancers for Igf2 in the minute mutant. Development 129, 1657-1668.

Davis, R. L., Weintraub, H. and Lassar, A. B. (1987). Expression of a single transfected cDNA converts fibroblasts to myoblasts. Cell 51, 987-1000.

DeChiara, T. M., Efstratiadis, A. and Robertson, E. J. (1990). A growthdeficiency phenotype in heterozygous mice carrying an insulin-like growth factor II gene disrupted by targeting. Nature 345, 78-80.

Engel, A. and Franzini-Armstrong, C. (2004). Myology. New York, NY: McGrawHill Professional.

Gabory, A., Ripoche, M. A., Le Digarcher, A., Watrin, F., Ziyyat, A., Forné, T., Jammes, H., Ainscough, J. F., Surani, M. A., Journot, L. et al. (2009). H19 acts as a trans regulator of the imprinted gene network controlling growth in mice. Development 136, 3413-3421.

Gabory, A., Jammes, H. and Dandolo, L. (2010). The H19 locus: role of an imprinted non-coding RNA in growth and development. BioEssays 32, 473 480

Gauthier-Rouviere, C., Vandromme, M., Tuil, D., Lautredou, N., Morris, M., Soulez, M., Kahn, A., Fernandez, A. and Lamb, N. (1996). Expression and activity of serum response factor is required for expression of the muscledetermining factor MyoD in both dividing and differentiating mouse $\mathrm{C} 2 \mathrm{C} 12$ myoblasts. Mol. Biol. Cell 7, 719-729.

Hagège, H., Klous, P., Braem, C., Splinter, E., Dekker, J., Cathala, G., de Laat, W. and Forné, T. (2007). Quantitative analysis of chromosome conformation capture assays (3C-qPCR). Nat. Protoc. 2, 1722-1733.

Hasty, P., Bradley, A., Morris, J. H., Edmondson, D. G., Venuti, J. M., Olson, E. N. and Klein, W. H. (1993). Muscle deficiency and neonatal death in mice with a targeted mutation in the myogenin gene. Nature $\mathbf{3 6 4}, 501-506$.

Inanlou, M. R. and Kablar, B. (2003). Abnormal development of the diaphragm in mdx:MyoD-/-(9th) embryos leads to pulmonary hypoplasia. Int. J. Dev. Biol. 47, 363-371

Inanlou, M. R., Dhillon, G. S., Belliveau, A. C., Reid, G. A., Ying, C., Rudnicki, M. A. and Kablar, B. (2003). A significant reduction of the diaphragm in mdx:MyoD-/-(9th) embryos suggests a role for MyoD in the diaphragm development. Dev. Biol. 261, 324-336.

Ishihara, K., Hatano, N., Furuumi, H., Kato, R., Iwaki, T., Miura, K., Jinno, Y. and Sasaki, H. (2000). Comparative genomic sequencing identifies novel tissue-specific enhancers and sequence elements for methylation-sensitive factors implicated in Igf2/H19 imprinting. Genome Res. 10, 664-671.

Kablar, B., Krastel, K., Ying, C., Asakura, A., Tapscott, S. J. and Rudnicki, M. A. (1997). MyoD and Myf-5 differentially regulate the development of limb versus trunk skeletal muscle. Development 124, 4729-4738. 
Kablar, B., Asakura, A., Krastel, K., Ying, C., May, L. L., Goldhamer, D. J. and Rudnicki, M. A. (1998). MyoD and Myf-5 define the specification of musculature of distinct embryonic origin. Biochem. Cell Biol. 76, 1079-1091.

Kablar, B., Krastel, K., Tajbakhsh, S. and Rudnicki, M. A. (2003). Myf5 and MyoD activation define independent myogenic compartments during embryonic development. Dev. Biol. 258, 307-318.

Kaffer, C. R., Grinberg, A. and Pfeifer, K. (2001). Regulatory mechanisms at the mouse Igf2/H19 locus. Mol. Cell. Biol. 21, 8189-8196.

Kaufman, M. (1992). The Atlas of Mouse Development. San Diego, CA: Academic Press.

L'honore, A., Lamb, N. J., Vandromme, M., Turowski, P., Carnac, G. and Fernandez, A. (2003). MyoD distal regulatory region contains an SRF binding CArG element required for MyoD expression in skeletal myoblasts and during muscle regeneration. Mol. Biol. Cell 14, 2151-2162.

Lake, B. D. and Wilson, J. (1975). Zebra body myopathy. Clinical, histochemical and ultrastructural studies. J. Neurol. Sci. 24, 437-446.

Leighton, P. A., Ingram, R. S., Eggenschwiler, J., Efstratiadis, A. and Tilghman, S. M. (1995). Disruption of imprinting caused by deletion of the H19 gene region in mice. Nature $\mathbf{3 7 5}, 34-39$.

Macharia, R., Otto, A., Valasek, P. and Patel, K. (2010). Neuromuscular junction morphology, fiber-type proportions, and satellite-cell proliferation rates are altered in MyoD(-/-) mice. Muscle Nerve 42, 38-52

Megeney, L. A., Kablar, B., Garrett, K., Anderson, J. E. and Rudnicki, M. A. (1996). MyoD is required for myogenic stem cell function in adult skeletal muscle. Genes Dev. 10, 1173-1183.

Merrick, D., Ting, T., Stadler, L. K. and Smith, J. (2007). A role for Insulin-like growth factor 2 in specification of the fast skeletal muscle fibre. BMC Dev. Biol. 7, 65 .

Montarras, D., Aurade, F., Johnson, T., Ilan, J., Gros, F. and Pinset, C. (1996). Autonomous differentiation in the mouse myogenic cell line, C2, involves a mutual positive control between insulin-like growth factor II and MyoD, operating as early as at the myoblast stage. J. Cell Sci. 109, 551-560.

Nabeshima, Y., Hanaoka, K., Hayasaka, M., Esumi, E., Li, S., Nonaka, I. and Nabeshima, Y. (1993). Myogenin gene disruption results in perinatal lethality because of severe muscle defect. Nature 364, 532-535.

Nowak, K. J., Ravenscroft, G. and Laing, N. G. (2013). Skeletal muscle a-actin diseases (actinopathies): pathology and mechanisms. Acta Neuropathol. 125, 19-32.

Pallafacchina, G., François, S., Regnault, B., Czarny, B., Dive, V., Cumano, A., Montarras, D. and Buckingham, M. (2010). An adult tissue-specific stem cell in its niche: a gene profiling analysis of in vivo quiescent and activated muscle satellite cells. Stem Cell Res. 4, 77-91.

Polesskaya, A., Naguibneva, I., Fritsch, L., Duquet, A., Ait-Si-Ali, S., Robin, P., Vervisch, A., Pritchard, L. L., Cole, P. and Harel-Bellan, A. (2001). CBP/p300 and muscle differentiation: no HAT, no muscle. EMBO J. 20, 68166825 .
Pownall, M. E., Gustafsson, M. K. and Emerson, C. P., Jr (2002). Myogenic regulatory factors and the specification of muscle progenitors in vertebrate embryos. Annu. Rev. Cell Dev. Biol. 18, 747-783.

Qiao, Y., Ma, N., Wang, X., Hui, Y., Li, F., Xiang, Y., Zhou, J., Zou, C., Jin, J., Lv, G. et al. (2011). MiR-483-5p controls angiogenesis in vitro and targets serum response factor. FEBS Lett. 585, 3095-3100.

Ren, H., Yin, P. and Duan, C. (2008). IGFBP-5 regulates muscle cell differentiation by binding to IGF-II and switching on the IGF-II auto-regulation loop. J. Cell Biol. 182, 979-991.

Ripoche, M. A., Kress, C., Poirier, F. and Dandolo, L. (1997). Deletion of the H19 transcription unit reveals the existence of a putative imprinting control element. Genes Dev. 11, 1596-1604.

Rotwein, P. (2003). Insulin-like growth factor action and skeletal muscle growth, an in vivo perspective. Growth Horm. IGFRes. 13, 303-305.

Rudnicki, M. A., Braun, T., Hinuma, S. and Jaenisch, R. (1992). Inactivation of MyoD in mice leads to up-regulation of the myogenic HLH gene Myf-5 and results in apparently normal muscle development. Cell 71, 383-390.

Rudnicki, M. A., Schnegelsberg, P. N., Stead, R. H., Braun, T., Arnold, H. H. and Jaenisch, R. (1993). MyoD or Myf-5 is required for the formation of skeletal muscle. Cell 75, 1351-1359.

Sambasivan, R. and Tajbakhsh, S. (2007). Skeletal muscle stem cell birth and properties. Semin. Cell Dev. Biol. 18, 870-882.

Schmitt, A., Guichard, J., Massé, J. M., Debili, N. and Cramer, E. M. (2001). Of mice and men: comparison of the ultrastructure of megakaryocytes and platelets. Exp. Hematol. 29, 1295-1302.

Smith, F. M., Garfield, A. S. and Ward, A. (2006). Regulation of growth and metabolism by imprinted genes. Cytogenet. Genome Res. 113, 279-291.

Smits, G., Mungall, A. J., Griffiths-Jones, S., Smith, P., Beury, D., Matthews, L., Rogers, J., Pask, A. J., Shaw, G., VandeBerg, J. L. et al. (2008).

Conservation of the H19 noncoding RNA and H19-IGF2 imprinting mechanism in therians. Nat. Genet. 40, 971-976.

Vandesompele, J., De Preter, K., Pattyn, F., Poppe, B., Van Roy, N., De Paepe, A. and Speleman, F. (2002). Accurate normalization of real-time quantitative RT-PCR data by geometric averaging of multiple internal control genes. Genome Biol. 3, RESEARCH0034.

Wilkin, F., Paquette, J., Ledru, E., Mamelin, C., Pollak, M. and Deal, C. L. (2000). H19 sense and antisense transgenes modify insulin-like growth factor-II mRNA levels. Eur. J. Biochem. 267, 4020-4027.

Wilson, E. M. and Rotwein, P. (2006). Control of MyoD function during initiation of muscle differentiation by an autocrine signaling pathway activated by insulin-like growth factor-II. J. Biol. Chem. 281, 29962-29971.

Woelfle, J., Chia, D. J., Massart-Schlesinger, M. B., Moyano, P. and Rotwein, P. (2005). Molecular physiology, pathology, and regulation of the growth hormone/insulin-like growth factor-l system. Pediatr. Nephrol. 20, 295-302.

Yoon, Y. S., Jeong, S., Rong, Q., Park, K. Y., Chung, J. H. and Pfeifer, K. (2007). Analysis of the H19ICR insulator. Mol. Cell. Biol. 27, 3499-3510. 